\title{
Lean Hospital: Der Patient im Zentrum
}

\section{Katharina V. Rüther-Wolf}

Dr. med., MBA, Universitätsspital Basel

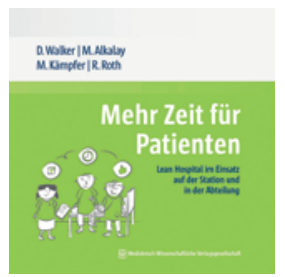

Korrespondenz: Dr. med. Katharina V. Rüther-Wolf, MBA Hebelstrasse 10 CH-4031 Basel

katharina.ruether[at]usb.ch
Walker, D.; Alkalay, M.; Kämpfer, M.; Roth, R.

Mehr Zeit für Patienten. Lean Hospital im Einsatz auf der Station und in der Abteilung.

Berlin: Medizinisch Wissenschaftliche Verlagsgesellschaft; 2017.

198 Seiten. 53 CHF.

ISBN 978-3-954-66310-1

Das Gesundheitswesen befindet sich in einem Strukturwandel. Schlankere Strukturen sind notwendig, um das Leistungsangebot und die Qualität aufrechtzuerhalten, die Selbstfinanzierung sicherzustellen und die Konkurrenzfähigkeit zu gewährleisten. Lean Hospital bietet unterschiedliche Mittel, eine schlanke operative Strategie erfolgreich umzusetzen und somit die Qualität, Produktivität und Mitarbeiterzufriedenheit und -engagement kontinuierlich weiterzuentwickeln.

Die Autoren zeigen auf, dass Lean als Denkweise gut zur Expertenorganisation Krankenhaus passt und unter anderem durch die Schaffung einer Kultur der kontinuierlichen Verbesserung einen neuen Standard in der stationären Gesundheitsversorgung setzt. Die Autoren stellen dem Leser die einzelnen Komponenten der Lean-Bettenstation ausführlich vor und bieten somit einen umfangreichen Überblick für all jene, die sich mit der Lean-Bettenstation auseinandersetzen. In zahlreichen praktischen Beispielen und Erfahrungsberichten wird in verständlicher Sprache beschrieben, wie gewisse Arbeitsweisen in der heutigen Komplexität unpraktisch und ineffizient sind. Durch die Be- trachtung der Abläufe aus der Sichtweise unterschiedliche Protagonisten werden im Buch Verschwendungen klar und unkompliziert dargestellt und analysiert. Überwiegend reaktive Arbeitsweisen verschiedener Berufsgruppen suggerieren zwar jedem einzelnen Bedeutung, machen aber eine Zusammenarbeit und $\mathrm{Ab}$ stimmung schwierig. Das Buch ist ein Plädoyer dafür, in der Organisation von Spitälern das zuzulassen, was in der Medizin selbstverständlich ist: Innovation und Fortschritt.

Lean Hospital stellt den Patienten ins Zentrum. Die Autoren zeigen auf, dass die Lean-Arbeitsweise eine grundlegende Philosophie der Veränderung bedeutet

Das Buch ist ein Plädoyer dafür, in der Organisation von Spitälern das zuzulassen, was in der Medizin selbstverständlich ist: Innovation und Fortschritt.

und ein langfristiger Prozess ist, der von der Führung unterstützt werden muss, um nachhaltig erfolgreich zu sein. Das Buch stellt diverse Taktiken zur Lösungsfindung und Anleitungen zur Umsetzung vor, um die Leistungsfähigkeit der Station zu steigern. Es geht darum, nicht sofort perfekt zu sein, aber stetig nach Perfektion zu streben.

Leider betrachtet das Buch nicht alle Bereiche des Krankenhauses. Gespannt warte ich daher auf ein entsprechendes Buch über die Implementierung von Lean Hospital im ambulanten Bereich sowie in den unterstützenden Bereichen eines Krankenhauses. 